\title{
The sun shadow calculation and analysis
}

\author{
Chai Jianeng \\ School of The North China Electric Power University,Baoding 071000,China \\ 1347757957@qq.com
}

Keywards: the length of the shadow,Control variable method.

\begin{abstract}
How to determine the location of the video is the important aspect of video data analysis and date taken, the sun's shadow positioning technology is based on analysising the sun's shadow change to determine the video shooting place and the date of video shooting.

Firs , through the literature to get the expression of the length of the shadow ,we find the longitude, latitude and date/time impact Angle of the sun, and then affect the length of the shadow. When we analysis the length of the shadow change rules of each parameter, we adopt the control variable method, and find that the length of the shadow basically don't change a lot with the growth of the year, with the increase of month and longitude, the length of the shadow gets shorter first, then gets longer, but with the increase of latitude the length of the shadow just gets longer. According to the expression of the length of the shadow We can get the curve of the impact of different parameters on the length of the shadow.
\end{abstract}

\section{Introduction}

First of all, we need to establish a mathematical model of the length of the shadow,and then analysis the length of the shadow change rules of each parameter. Based on consulting a large number of information, through the solar declination Angle, True solar time, Mean solar time,local time , true local time , Angle, local latitude and longitude, finally we get the sine value expression of the solar altitude Angle. According to trigonometric function we get the length of the shadow, the length of the rods and the expression of the solar altitude Angle.

According to the expression of the solar altitude Angle, through the control variable method, we make sure of a certain variable changes, other variables remain the same, then get the length of shadow about the change rule of each variable. Finilly we draw the length of shadow of the 3 meters high straight rod curveon October 22, 2015, Beijing time between 9:00-15:00 Tiananmen square (39 degrees north latitude 54 minutes and 26 seconds, east longitude 116 degrees 23 minutes 29 seconds), since data, longitude and latitude is certain, only the time of day in the change, and we can get the calculation curves.

\section{Models for the length of shadow}

\subsection{Assumptions \&Symbols,Definitions}

\section{- Assumptions}

$>$ We assume that the earth is a sphere.

$>$ We assume that do not consider the sun through the atmosphere refraction occur, the surface of the sun Angle, mountain block, altitude, we assume the light from the sun is parallel light.

$>$ We assume that the earth's rotation is uniform. 
- Symbols\&Definitions

\begin{tabular}{|c|l|c|l|}
\hline$E_{D}$ & The sun declination Angle & $H_{A}$ & The hour angle \\
\hline$E_{t}$ & The equation of time & $l_{a}$ & The local latitude \\
\hline$S_{1}$ & The True solar time & $h$ & $\begin{array}{l}\text { The Sine value of the solar altitude } \\
\text { Angle }\end{array}$ \\
\hline$S_{d}$ & The local Time & $d_{a}$ & The day angel \\
\hline$S_{2}$ & The true local time & C & The solar altitude angle \\
\hline$S$ & The Mean solar time & $l_{o}$ & The Local longitude \\
\hline
\end{tabular}

\subsection{Model construction}

Through geographical knowledge and mathematical formula derivation ${ }^{[1]}$, we get the formula of the solar altitude Angle,then we deduce the formula of the length of the shadow.

2.2.1 The calculation of the length of the shadow

1.Through geographical knowledge,we can get the serial number of dates in years which is No $=79.6764+0.2422(y-1985)-7$.

2. $d_{a}=2 \times \pi \times t / 365.2422$, where $\mathrm{t}=\mathrm{N}-\mathrm{No}$.

3.The calculate of the solar declination Angle ${ }^{[4]}$ :

$E_{D}=0.3723+23.2567 \times \sin \left(d_{a}\right)+0.114 \times \sin \left(2 d_{a}\right)-0.1712 \times \sin \left(3 d_{a}\right)-0.758 \times \cos \left(d_{a}\right)+0.3656 \times \cos \left(2 d_{a}\right)$

$+0.0201 \times \cos \left(3 d_{a}\right)$

calculation of the equation of time:

$E_{t}=0.0028-1.9857 \times \sin \left(d_{a}\right)+9.9059 \times \sin \left(2 d_{a}\right)-7.0924 \times \cos \left(d_{a}\right)-0.6882 \times \cos \left(2 d_{a}\right)$, when we get the equation of time,we can obtain the True solar time $s_{1}=S+E_{t} / 60$, the true local time $\boldsymbol{S}_{2}=\boldsymbol{S}_{d}+E_{t} / 60$,the local Time ${ }_{S_{d}=} s-\left(120-l_{0}\right) \times 4 / 60$ and the hour angle $s_{d}=\left(s_{2}-12\right) \times 15 \times \pi / 180$.

5.The calculation of the solar altitude angle:

$$
h=\sin \left(l_{a}\right) \times \sin \left(E_{d} \times \pi / 180\right)+\cos \left(l_{a}\right) \times \cos \left(E_{d} \times \pi / 180\right) \times \cos \left(H_{A}\right), c=\arcsin h
$$

6.The calculation of the length of the shadow: $l=x / \tan c$, where $\mathrm{x}$ is the length of the rod.

\subsection{Result and analysis}

2.3.1 The Tiananmen square rod's length of shadow change curve ${ }^{[2]}$

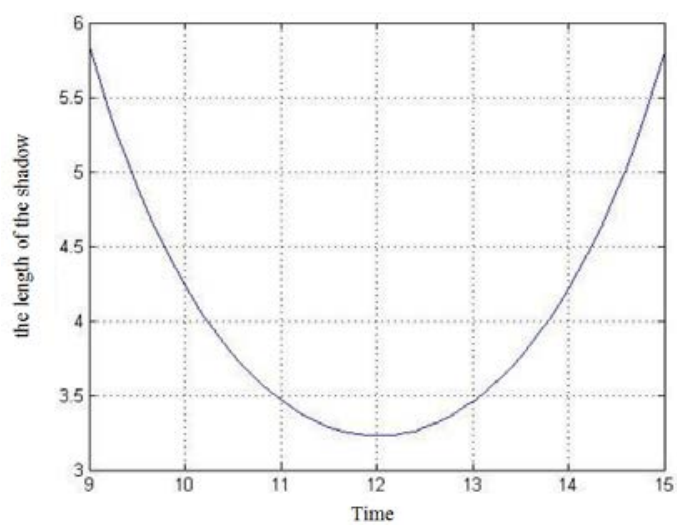

Fig 1 the length of the shadow

From the figure,we can see that the length of the shadow is shortest in $12 \mathrm{am}$, Angle of the sun at this time is the largest.

2.3.2 The influence year change

We draw the length of shadow of the 3 meters high straight rod curve on October 22, 1980-1990, 
Beijing time between 12:00 Tiananmen square (39 degrees north latitude 54 minutes and 26 seconds, east longitude 116 degrees 23 minutes 29 seconds), we make sure of a year variable changes, other variables remain the same.

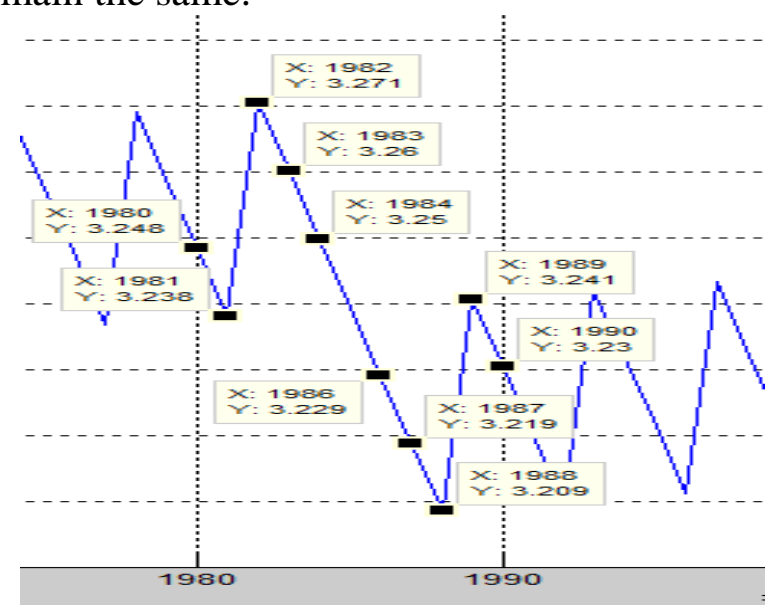

Fig 2 the shadow length along with the change of year

From fig 2, we can konw that he length of the shadow basically don't change a lot with the growth of the year with the change of year

2.3.4 The influence of month and longitude change

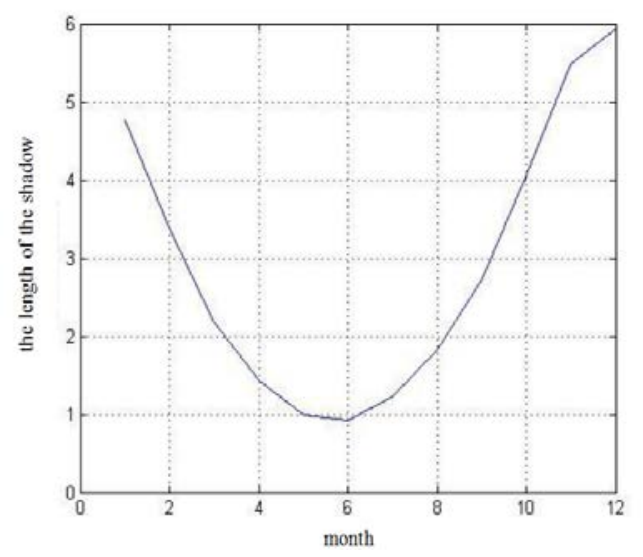

Fig 3 the influence of month change

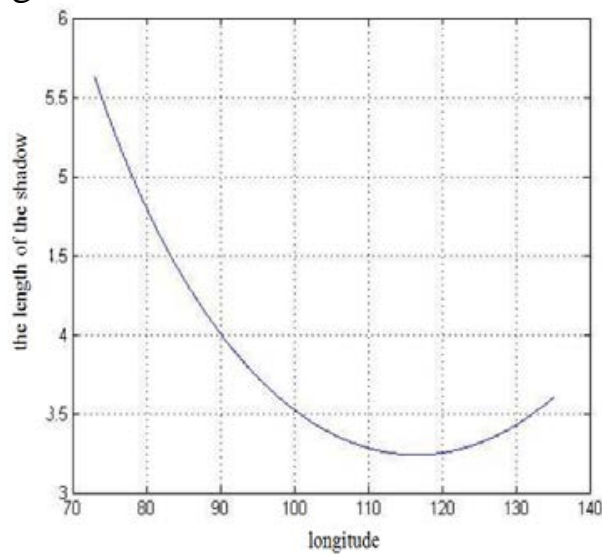

Fig 4 the influence of longitude change

With the increase of month and longitude, the length of the shadow gets shorter first, then gets longer.

2.3.2 The influence of latitude change

We draw the length of shadow of the 3 meters high straight rod curve on October 22, 2015, Beijing time between 12:00 Tiananmen square (0-60 degrees north latitude 54 minutes and 26 seconds, east longitude 116 degrees 23 minutes 29 seconds) ,we make sure of a latitude variable changes, other variables remain the same.

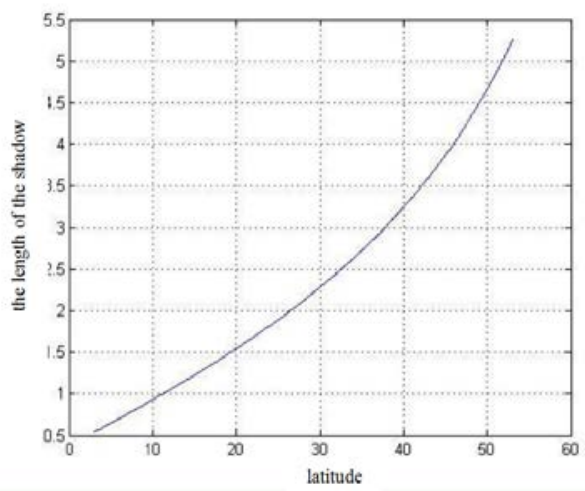

Fig 5 The influence of latitude change

From fig 5,we can know that with the increase of latitude the length of the shadow just gets 
longer.

\section{Conclusions}

When we analysis the length of the shadow change rules of each parameter, we adopt the control variable method, and find that the length of the shadow basically don't change a lot with the growth of the year, with the increase of month and longitude, the length of the shadow gets shorter first, then gets longer, but with the increase of latitude the length of the shadow just gets longer.

\section{Reference}

[1]Zhang Wenhua ,The Method of Sun Shadow Ratio Calculation and its Influence on The Photovoltaic Array Layout[J], Technology and products,2011

[2]Si Shoukui,Sun Xijing,Mathematical Modeling,National Defense Industry press,June 2014,

[3] IEA. Compared Assessment of Selected Environment Indicators of Photovoltaic Electricity in OECD Cities[R]. Report IEA-PVPS Task 10, 2006.

[4]http://baike.baidu.com/view/53842.htm?fromtitle=\%E5\%A4\%AA\%E9\%98\%B3\%E8\%B5\%A4 \%Е7\%BA\%AC\%E8\%A7\%92\&type=syn 